\title{
Buchbesprechungen
}

\section{Ulrich Sollmann (2018). Begegnungen im Reich der Mitte. Mit psychologischem Blick unterwegs in China}

\section{Gießen, Psychosozial-Verlag. 280 Seiten. 24,90 EUR}

Psychotherapie-Wissenschaft 8 (2) 89-90 2018

www.psychotherapie-wissenschaft.info

CC BY-NC-ND

https://doi.org/10.30820/8243.18

Um gleich vorweg auf die vom Autor im Epilog geäusserte Hoffnung zu antworten: Ja, meine Neugierde und Lust auf weitere eigene ethnologische Wanderungen in China wurden im Laufe der Lektüre dieses Buches belebt.

Als einer, der selbst seit einigen Jahren nach China reist und dort lehrt, war ich sehr gespannt auf dieses Buch eines Fachkollegen und habe vieles gefunden, was mir von meinen eigenen Eindrücken und Erlebnissen her bekannt vorkam, manches aber auch, wo ich dachte: So habe ich noch gar nicht darüber nachgedacht oder es so betrachtet. Und natürlich auch manches, das mich eher irritierte. Doch der Reihe nach.

Ulrich Sollmann ist Körpertherapeut und über die «Deutsch-Chinesische Akademie für Psychotherapie» erstmals 2013 aus beruflichen Gründen nach China gereist. Seither besucht er das Land regelmässig und verbindet seine beruflichen Tätigkeiten mit privaten Erkundigungen über das Leben in China.

In diesem Buch schildert er, wie er sich in dieser ihm fremden Kultur bewegt und zurechtzufinden versucht. Er sucht dabei nach einer ethnologischen Haltung, aus der er möglichst vorurteilsfrei phänomenologisch beobachtet und das, was er sieht und erlebt, auf sich wirken lässt, um erst dann nach Erklärungen und Interpretationen zu suchen, um zu verstehen. Er versteht das als eine Grundhaltung, die er auch als professionelle Naivität bezeichnet, die erst entwickelt werden will und Grundvoraussetzung ist, einer fremden Kultur begegnen zu können. Er sieht seine «ethnologischen Wanderungen» als Aktionsforschung. Im Sinne dieser Forschungstradition wird er natürlich auch als Beobachtender ein Teilnehmer der beobachteten Situationen und Teil des Feldes. Das beeinflusst ihn und die übrigen an der Situation Beteiligten. Um nicht draussen zu bleiben, aber auch nicht vereinnahmt zu werden, spricht er von einer Juxtaposition, die einzunehmen ist, um einerseits mit dem Eigenen in Verbindung zu bleiben und andererseits sich auf das Fremde einlassen zu können. Die Juxtaposition ist eine dritte Position, aus der man mit etwas Distanz beides im Auge halten kann: das Eigene und das Fremde. Im Eintauchen in eine fremde Zivilisation entsteht erst einmal ein Orientierungsvakuum. Die mitgebrachten Verhaltensweisen der eigenen Kultur greifen nicht und die fremden Regeln kennt man noch nicht.
Sollmann berichtet über seine Erlebnisse in einem narrativen Stil, dem man gut folgen kann, und offenbart dabei auch viel Persönliches. Sein Buch erlaubt es, ihm als Mensch und als Psychotherapeut, Kursleiter oder Berater in Firmen über die Schulter zu sehen und teilzuhaben an seinen Erlebnissen in unterschiedlichsten Begegnungssituationen. Als Körpertherapeut gibt er der Beobachtung des körperlichen und nonverbalen Ausdrucks viel Aufmerksamkeit. In seiner Art, Erlebtes zu reflektieren und dabei auf seine Gegenübertragungsreaktionen zu achten, glaubt man, einem Psychoanalytiker zu folgen. In der Betonung auf das Erleben im Hier und Jetzt erkennt man einen humanistischen Psychologen.

Das Buch ist in mehrere Kapitel eingeteilt, die einen Aufbau vom sich Herantasten in ersten Begegnungen und Erlebnissen hin zur Beschreibung der aktuellen Lebenswelt in China, Beschreibungen, wie sich die Gesellschaft organisiert und funktioniert, haben. Er beschreibt unterschiedliche soziale Räume und die Funktion von Mauern, die schützen und zugleich ein unterschiedliches Sozialverhalten ermöglichen. Drei typische Begegnungsräume mit ihrer jeweils eigenen charakteristischen Art der Selbstregulation werden beschrieben: der Individualraum, der Situationsraum und der Übergangsraum als Spannungsfeld zwischen zwei Räumen. Dem Individualraum ordnet er ein Verhalten zu, wo man ganz auf sich selbst ausgerichtet ist, sei es, indem man alleine ist oder mit sich selbst verbunden in der Öffentlichkeit (oft in Pärken) tanzt oder sich in Gesellschaft so verhält, als wäre man allein. Situationsräume sieht er in Gebäuden, Siedlungen, oft von Mauern umgeben, oder in sozialen kollektiven Räumen, die durch ethnische Zugehörigkeit entstehen, oder in Situationen wie Meetings, zum Beispiel in Restaurants, in denen ein jeweils unterschiedliches Verhalten derselben Person zu beobachten ist. Situationsräume führen zu Rollenverteilungen, die das Verhalten beeinflussen. Die Wechsel zwischen den Situationsräumen können abrupt und überraschend erfolgen, auch solche zwischen Individual- und Situationsräumen, was besondere Fähigkeiten für den Umgang mit diesen Wechseln erfordert. In den Übergangsräumen befindet man sich in einem Spannungsfeld bei Wechseln zwischen verschiedenen Situationsräumen oder dem Individualraum. Der Autor schildert diese Räume anhand anschaulicher Beispiele aus seinen Begegnungen.

Ein grösseres Kapitel widmet er der Beschreibung einer besonderen kulturellen Kompetenz, die er den Chinesen attestiert: Die sogenannte Brückenkompetenz. Sie ist mit der Ambiguitätstoleranz vergleichbar, mit der Fähigkeit, Spannungen in der Schwebe zu halten und mit widersprüchlichen Rollenerwartungen in unterschiedlichen Situationsräumen umzugehen. Auf dem Hintergrund traditioneller chinesischer Werte, speziell von Konfuzianismus und Legalismus, versteht er die Brückenkompetenz 
als Fertigkeit, die tief in der Geschichte Chinas und der darin vereinigten verschiedenen Kulturen verankert ist. Die Brückenkompetenz erlaubt die Entwicklung eines Sensoriums, wann und wo man etwa kritische Diskussionen zur chinesischen Politik führen kann und darf und wo und wann welche Grenzen zu respektieren sind.

In einem kurzen historischen Abriss erinnert er an den ersten Kaiser Chinas, Qin Shi Huang, der vor 2000 Jahren die sieben chinesischen Reiche zu einem geformt und geeint hat. Dieser nutzte drei strategische Massnahmen, die die chinesische Gesellschaft, Kultur und Politik bis heute prägen: Er schuf ganz unterschiedliche gesellschaftliche und organisationale Räume, die mit klaren Vorgaben oder auch durch Mauern begrenzt wurden. Innerhalb dieser Räume erlaubte er zweitens, dass man sich so verhalten konnte, wie man wollte. Ausser: Die Erhebung der Stimme gegen den Kaiser oder das politische System - das wurde mit der Todesstrafe geahndet. Er verband das Land durch den Bau eines landesweiten Verkehrsnetzes von Strassen und Kanälen und vereinheitlichte die Währung und die Gewichte. Dritte Massnahme war die Philosophie des Legalismus, ein bereits bestehendes, breites Gesetzessystem, das das Kollektiv über den Einzelnen stellte. Durchgesetzt wurde die Macht mittels Belohnung und Bestrafung. Der Einfluss dieses Systems wirkt in der Tat bis heute nach und hat in der ereignisreichen Geschichte Chinas keine wirkliche Veränderung erfahren. Umso mehr interkulturelle Reibungsfläche entsteht mit der Öffnung zu westlichen Gesellschaftssystemen, wo mit der Verankerung in der Philosophie des Liberalismus der Einzelne über das Kollektiv gestellt wird.

Um damit umgehen zu können, ist die in der chinesischen Kultur entwickelte Brückenkompetenz sehr wertvoll, für Westler aber oft verwirrend und nicht leicht verstehbar.

Das Buch gibt einen eindrucksvollen Einblick in viele Aspekte der chinesischen Gesellschaft. Es beschreibt auch die Problematik, dass viele Westler ihr Interesse an China darin sehen, die Chinesen anders zu sozialisieren, sie als noch etwas im Rückstand befindliche Kultur zu sehen, die man nach westlichem Bild emanzipieren müsste. Sie sehen sich als Helfer oder Erlöser und die Chinesen als Hilfsbedürftige und schaffen so eine asymmetrische Beziehungshierarchie, auf die Chinesen mit Recht aversiv reagieren. Dieser Falle versucht der Autor mit seiner ethnologischen Grundhaltung zu entkommen. Doch da und dort holt sie ihn selbst ein. Manche Stellen und Interpretationen sind nicht frei von deutschen Wertungen, etwa wenn er wiederholt chinesische Verhaltensweisen als kindlich bezeichnet, als zu wenig entwickelt. So sehr der Autor in den Schilderungen Chinas und des Verhaltens der Chinesen sorgfältig die kulturellen Hintergründe in ihrer geschichtlichen und philosophischen Dimension zu beachten versucht, so deutlicher wird, dass das Gegenstück, die Selbstreflexion darüber, fehlt, wie sich Begegnungen mit ihm als Deutschem mit der eigenen biografischen und kollektiven Geschichte und philosophischen Verankerung auf das Erleben und Erkennen des Fremden auswirken und die Situationen mitprägen. Ich weiss - ein hoher
Anspruch, den der Autor aber mit seinem Bezug zur Ethnologie selbst an sich stellt. Keine moderne ethnologische Forschung darf diese Selbstreflexion der eigenen kulturellen Prägung des Forschers und dessen Impact auf das Forschungsprojekt vernachlässigen. In Ansätzen berücksichtigt Sollmann dies durchaus, aber leider nicht konsequent genug.

Der Autor versteht seine geschilderten Eindrücke über die Chinesen als seine subjektiven Eindrücke, die keine Objektivität beanspruchen. Er schildert gar eine Sequenz, wo ein Chinese zu ihm sagt, er solle den Anspruch, über «die Chinesen» schreiben zu wollen, aufgeben, denn «die Chinesen» gäbe es nicht. Dennoch tendiert er immer wieder sehr zu verallgemeinernden Aussagen über «die Chinesen», gar auf derselben Buchseite. Die Legitimation, von subjektiven (von der eigenen Biografie und Kultur geprägten) Eindrücken zu objektivierenden verallgemeinernden Aussagen zu kommen, wird nicht erläutert. Schade. Da hätte ein inhaltlich strengeres Lektorat wohl helfen können, Widersprüche zwischen dem eigenen Anspruch und wie dieser eingelöst wird, zu bereinigen.

An manchen Stellen ist auch zu fragen, ob solche Verallgemeinerungen wirklich typisch für «die Chinesen» sind oder ob man nicht zu ähnlichen Aussagen kommen würde, wenn man «die Europäer», die «Schweizer» oder «die Deutschen» einfügen würde. Das ist übrigens eine gute Übung, um selbstreflexiv Vorurteile, Projektionen und Gegenübertragungsphänomene zu erkennen. Fachliche Streitdiskussionen könnten sich auch im Umgang mit Begrifflichkeiten ergeben, etwa wenn er den ganzen Körper als ein Sinnesorgan bezeichnet. Oder wenn er von einem «ethnologischen Welpenschutz» spricht.

Dennoch: Ich finde dieses Buch sehr lesenswert, reich, vielfältig und anregend für Menschen und PsychotherapeutInnen, die privat oder beruflich mit Menschen aus anderen Kulturen zu tun haben, insbesondere mit Menschen aus China.

Die Aufgabe einer Selbstreflexion über die WertePrägungen aus der eigenen Kultur und wie sich diese in der Begegnung mit Angehörigen aus einer fremden Kultur treffen oder in Konflikt geraten, gehört eh zur fortlaufenden Aufgabe eines jeden Lesenden und/oder Schreibenden sowie aller EthnologInnen und PsychotherapeutInnen, die sich auf die «Anderen» einlassen.

Peter Schulthess 